\title{
Inhibition of PTEN activity promotes IB4-positive sensory neuronal axon growth
}

Li-Yu Zhou ${ }^{1 \#}$, Feng Han ${ }^{1 \#}$, Shi-Bin $\mathrm{Qi}^{1}$, Jin-Jin Ma ${ }^{1}$, Yan-Xia Ma ${ }^{1}$, Hong-Cheng Zhang ${ }^{1}$, Ji-Le, Xie ${ }^{1}$, Xin-Ya, Fu ${ }^{1}$, Jian-Quan $\mathrm{Chen}^{1}$, Bin $\mathrm{Li}^{1}$, Hui-Lin Yang ${ }^{1 *}$, Feng Zhou ${ }^{{ }^{*}}$ and Saijilafu ${ }^{*}$

${ }^{1}$ Department of Orthopaedics, The First Affiliated Hospital of Soochow University, Orthopedic Institute, Soochow University, Suzhou, Jiangsu, P. R. China.

\# These authors contributed equally to this work

*Correspondence: Saijilafu, MD, Ph.D.

Phone: 0512-6778-0762

Email: saijilafu@suda.edu.cn

Running title: PTEN and IB4-positive neuronal axon growth

\section{Author Contributions}

LY Z, F H, SB Q, JJ M, YX M, HC Z, JL X, XY F, JQ C, B L, HL Y, F Z, and Saijilafu designed the experiment. LY Z, F H, and SB Q performed the experiments. LY Z, F H, and Saijilafu co-wrote the manuscript.

Funding Statement: This work was supported by a grant from the National Natural Science Foundation of China (No. 81571189 and No. 81772353 to Saijilafu), National Key Research and Development Program (No. 2016YFC1100203), An Innovation and Entrepreneurship Program of Jiangsu Province, and A Priority Academic Program Development of Jiangsu Higher Education Institutions.

Conflict of Interest Statement: The authors declare that there is no competing interest.

Data Accessibility Statement: The data that support the findings of this study are available from the corresponding author upon reasonable request. 

58 59

\section{Abstract:}

Traumatic nerve injuries have become a common clinical problem, and axon regeneration is a critical process in the successful functional recovery of the injured nervous system. In this study, we found that peripheral axotomy reduce total PTEN expression in adult sensory neurons, however, it did not alter the expression level of PTEN in IB4-positive sensory neurons. Additionally, our results indicate that the artificial inhibition of PTEN markedly promotes adult sensory axon regeneration, including IB4-positive neuronal axon growth. Thus, our results provide strong evidence that PTEN is a prominent repressor of adult sensory axon regeneration, especially in IB4-positive neurons.

Key words: sensory neuron; axon regeneration; PTEN; IB4-positive neurons

(1)

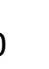

1

5

\section{7}




\section{Introduction}

With the rapid rise in traffic accidents, sports participation, and construction work, the incidence of nerve injury is increasing each year. However, functional recovery from nerve injury is often unsatisfactory as many patients suffer from permanent complications, such as paralysis. In the nervous system, axons are the main transmission pathway of electric impulses between two nerve cells, or between a nerve cell and a downstream effector cell. Thus, axon regeneration is a critical process for the successful functional recovery of the injured nervous system. However, there are two major factors that inhibit axonal regeneration. The first is the inhibitory external environment, including mechanical barriers like a fibrosis scar. The second is the diminished axon regeneration ability of mature neurons (Liu et al., 2010; Park et al., 2010). Previous literature has demonstrated that the manipulation of the inhibitory microenvironment can promote axonal regeneration, but the effects are limited (Lee et al., 2010). Therefore, today, enhancing the intrinsic axon regeneration ability of mature neurons has become a new strategy to promote the growth of injured axons. To date, recent studies have identified that the PTEN gene is a critical regulator of the intrinsic axon regeneration ability of mature neurons, and that PTEN knockout significantly promotes axon generation in the central nervous system (CNS) (Du et al., 2015; Kurimoto et al., 2010; Liu et al., 2010; Ning et al., 2010; Ohtake et al., 2015; Park et al., 2010).

It has been reported that mammalian dorsal root ganglia (DRG) contain several subpopulations of neurons that possess different characteristics. For example, Griffonia simplicifolia isolectin B4 (IB4) can recognize the subpopulation of neurons that expresses the receptors for glial cell line-derived neurotrophic factor (Bennett et al., 1998; Tucker et al., 
2006). Additionally, IB4-labeled neurons are difficult to regenerate axon after injury, and even the forced overexpression of axon growth promoting molecules, such as $\alpha 7 \beta 1$-integrin and GAP43, failed to enhance the axon growth of IB4-labeled neurons (Leclere et al., 2007). These findings indicate that IB4-labeled DRG neurons lack the intrinsic ability to regenerate axons following injury. Interestingly, a recent study found that IB4-labeled neurons usually have an elevated expression of PTEN protein (Christie et al., 2010). Therefore, we hypothesized that the PTEN gene is one of the crucial inhibitors of IB4-positive (IB4 ${ }^{+}$) neuronal axon regeneration. Thus, the suppression of PTEN activity may increase the intrinsic axon growth ability of $\mathrm{IB}^{+}{ }^{+}$neurons, and further promote its axon regeneration after injury.

In the present study, we found that the expression of total PTEN in adult sensory neurons is down-regulated by peripheral axon injury, and inhibition of PTEN promotes its axon regeneration. However, we also found that $\mathrm{IB}^{+}{ }^{+}$neurons possess high level of PTEN, and peripheral axotomy did not alter the expression level of PTEN in IB4 ${ }^{+}$neurons. Our results further indicate that artificial inhibition of PTEN markedly promotes IB4 ${ }^{+}$neuronal axon growth in vitro. Thus, here we provide strong evidence that PTEN is a prominent repressor of adult sensory axon regeneration, especially in IB $4^{+}$neurons.

\section{Methods and Materials}

\section{Animals and surgical procedures}

All animals (adult ICR mice, PTEN ${ }^{\text {flox/flox }}$ mice, and Advilin-Cre mice, from 8-10 weeks of age) were handled according to the guidelines of the Institutional Animal Care and Use Committee of Soochow University. Advilin-Cre and PTEN ${ }^{\text {flox/flox }}$ mice were bred together to 
generate DRG sensory neuronal specific PTEN knockout mice. The PTEN ${ }^{\text {flox/flox }}$ mice were obtained from Prof Liu Yaobo (Soochow University), Advilin-Cre mice were obtained from Zhou Fengquan (Johns Hopkins University). Surgical procedures were performed under intraperitoneal anesthesia with ketamine $(100 \mathrm{mg} / \mathrm{kg})$ and xylazine $(10 \mathrm{mg} / \mathrm{kg})$. To create the sciatic nerve injury, the skin was disinfected, and scissors were used to make a longitudinal incision of approximately $1 \mathrm{~cm}$. The muscles were separated, and the sciatic nerve was exposed. The sciatic nerve was then transected, and the skin incision was closed. Mice that received a sham surgery were used as controls.

\section{Reagents and antibodies}

The neuron-specific class III $\beta$-tubulin mouse mAb (Tuj1, 1:1500) antibody was purchased from Covance. The PTEN antibody was purchased from Santa Cruz Biotechnology. Secondary antibodies conjugated with Alexa fluorophores 488 or 568 were directed against the IgGs of the primary antibody species (1:1000; Invitrogen). The Alexa Fluor 488-conjugated Isolectin IB4 was from life technology. The SF1670 and BPV were purchased from Selleck. The PTEN primer (forward: 5'-CTCCTCTACTCCATTCTTCCC-3'; reverse: 5'-ACTCCCACCAATGAACAAAC-3') was from Gene Pharma (GenePharma Co.; Shanghai, China).

\section{Adult sensory neuronal culture}

The Lumber 4 (L4) and L5 DRG were dissected out and treated with $2 \mathrm{ml} 0.1 \%$ collagenase for $90 \mathrm{~min}$ at $37.0{ }^{\circ} \mathrm{C}$. Then, the samples were treated again with $1 \mathrm{ml}$ Tryple Express for $20 \mathrm{~min}$ at $37.0^{\circ} \mathrm{C}$. Tryple Express was neutralized with culture medium (the Minimum Essential Medium supplemented with 5\% fetal bovine serum, 
$1 \times$ Penicillin/Streptomycin solution). Then, the DRGs were dissociated with a $1000 \mu 1$ pipette tip using the culture medium. The dissociated cells were cultured on poly-D-lysine $(100 \mu \mathrm{g} / \mathrm{ml}$, Sigma) and laminin (10 $\mu \mathrm{g} / \mathrm{ml}$, Sigma) coated coverslips in $24-w e l l$ plates. The isolated neurons were allowed to grow axons for 3 days at $37^{\circ} \mathrm{C}$ in a $\mathrm{CO}_{2}$-humidified incubator. During cell culture, we administered SF1670 (1 nM or $10 \mathrm{nM})$, or $200 \mathrm{nM} \mathrm{BPV}$ to the culture medium. As a control, the same volume of DMSO was added to the culture medium.

\section{Axon length analysis}

All images were photographed using AxioVision 4.7 software (Carl Zeiss MicroImaging, Inc.). The longest axons of one hundred randomly selected neurons in each experimental condition were traced and measured manually using the "measure/curve" application of AxioVision 4.7 software. Average axon lengths were calculated from three separate experiments in each condition.

\section{Immunofluorescence staining}

The samples were fixed in 4\% PFA, and blocked with $2 \%$ BSA. DRG tissues were cut into slices of 14 microns and pasted on gelatin-coated glass slides. The primary antibody was applied for 24 hour at $4^{\circ} \mathrm{C}$, then washed with $3 \%$ TPBS and incubated with the secondary antibodies for 1 hour at room temperature. Afterwards, tissue sections were incubated with Hoechst (1:2000) for $20 \mathrm{~min}$ at room temperature. For immunofluorescence staining of IB4, Alexa Fluor 488-conjugated Isolectin IB4 was applied overnight at $4^{\circ} \mathrm{C}$, and then washed with 3\% TPBS and mounted with a custom-made MOWIOL mounting media.

\section{Western blots}

The proteins were extracted with RIPA buffer, and the protein concentrations were 
measured using Bio-Rad DC Protein Assay. The protein samples were loaded into SDS-PAGE gels, and then transferred to polyvinylidene fluoride membranes (PVDF). The PVDF membranes were incubated with the corresponding primary antibodies overnight at $4{ }^{\circ} \mathrm{C}$, and then with the secondary antibodies for 2 hours at room temperature. Protein bands were visualized using Pierce ${ }^{\mathrm{TM}}$ ECL Western Blotting Substrate, and the densities of protein bands from three independent experiments were quantified using Image $\mathbf{J}$ software (NIH, Bethesda, MD, USA).

\section{qRT-PCR}

Trizol reagent was used to extract RNA from the DRG tissue. RNA was treated with reverse transcription kits and processed for cDNA synthesis. PCR primers were purchased from Sangon Biotech. The PCR products were labeled using the CFX96 ${ }^{\mathrm{TM}}$ real-time PCR detection system (Bio-Rad). We used $18 \mathrm{~S}$ as a housekeeping gene.

The following primer sequences were used:

PTEN-F: 5'-CTCCTCTACTCCATTCTTCCC-3'

PTEN-R: 5'-ACTCCCACCAATGAACAAAC-3'

18S-F: 5-TCCCTAGTGATCCCCGAGAAGT-3'

18S-R: 5-CCCTTAATGGCAGTGATAGCGA-3'

\section{In vivo electroporation of adult DRG neurons}

Under deep anesthesia, the left L4-L5 DRGs of adult PTEN knockout mice were exposed, and $1.0 \mu 1$ of EGFP plasmid was micro-injected into the DRG tissue. The PTEN ${ }^{\text {flox/flox }}$ mice were used as the control group. After microinjection, the DRG was electroporated using a tweezer-like electrode $(\varnothing 1.0 \mathrm{~mm})$ and an ECM830 Porator for five pulses $(35 \mathrm{~V}, 15 \mathrm{~ms}$ 
duration, $950 \mathrm{~ms}$ interval). The animals were then allowed to recover. Two days later, the ipsilateral sciatic nerve was exposed, and the crush injury was made using \#5 fine forceps. An 11-0 nylon suture was used to mark the crush site. After another three days, the animals were perfused transcardially with 4\% PFA, and the entire sciatic nerve was dissected and removed. The lengths of all EGFP-positive axons were measured from the marking suture to the distal axon tip.

\section{Statistics}

Data are presented as mean \pm S.E.M. Two-tailed Student's t-tests were used to compare the different experimental conditions. $P$-values of $P<0.05$ were considered statistically significant $(* P<0.05 ; * * P<0.01 ; * * * P<0.001)$.

\section{Results}

Decreased expression of PTEN in adult sensory neurons following sciatic nerve

\section{transection}

Firstly, we found that, after sciatic nerve transection, the expression of total PTEN in L4/5 DRG neurons was significantly down-regulated compared to the control (Fig. 1a, b).

Secondly, the PTEN mRNA level in the DRG neurons also decreased following the sciatic nerve injury (Fig. 1c), and this finding was consistent with the observed changes in protein levels. It has been reported that PTEN is an important inhibitor of the intrinsic axon growth ability of mature CNS neurons. Furthermore, the genetic knockout of the PTEN gene can significantly promote axon regeneration in the CNS (Bhagat et al., 2014; Danilov and Steward, 
2015). However, the precise functional role of PTEN in the process of peripheral nervous system axon regeneration is still unknown. Using an in vitro adult peripheral sensory neuronal culture model, we found that pharmacological inhibition of PTEN activity promoted the axon growth of peripheral sensory neurons (Fig. 2a, b). Inhibition of PTEN markedly increased phospho-S6 expression in the cultured DRG neurons (Fig. 2c). Phospho-S6 is a well-known downstream target of the PTEN pathway. This result indicates that our drug concentration is enough to inhibit PTEN activity in the cultured DRG neurons. Considering the side effects of small chemical molecules, we used PTEN knockout mice to investigate their functional roles on peripheral axon regeneration. We consistently found that the axon length in the PTEN knockout mice was significantly longer than in the wild-type mice (Fig. 2d, e). Furthermore, the genetic knockout of PTEN also markedly promoted in vivo sciatic nerve axon regeneration (Fig. 2f, g). Taken together, our results indicate that the inhibition of PTEN activity promotes axon growth in adult sensory neurons in vitro and in vivo.

\section{IB4 $^{+}$neurons have a higher PTEN expression level than IB4- neurons, and PTEN}

\section{expression was not significantly decreased by sciatic nerve transection.}

Adult sensory neurons can be divided into two subpopulations according to their IB4 binding ability, referred to as IB4 ${ }^{+}$and IB4-negative (IB4 ${ }^{-}$) neurons. $\mathrm{IB}^{+}$neurons have very limited axon regeneration ability, and high levels of expression of PTEN protein. In agreement with previously published literature (Christie et al., 2010), our immunofluorescence staining results revealed that PTEN expression in the IB4 ${ }^{+}$neurons was significantly higher than in the IB4- neurons (Fig. 3a). Additionally, we found that the average axon length of the IB4 ${ }^{+}$neurons was significantly shorter than the IB4- neurons (Fig. 3b, c). 
Previous findings have demonstrated that peripheral axotomy can activate the intrinsic axon regeneration ability of adult sensory neurons (Smith and Skene, 1997). For this reason, we investigated the effects of peripheral axotomy on PTEN expression in IB4 ${ }^{+}$neurons. We found that peripheral axotomy did not affect the expression level of PTEN proteins in the $\mathrm{IB}^{+}{ }^{+}$neurons (Fig. 4a). Furthermore, axon growth from the $\mathrm{IB}^{+}{ }^{+}$neurons was not promoted by peripheral axotomy (Fig. 4b, c). However, we observed that peripheral axotomy significantly promote axon regeneration in IB4- neurons (Fig. 4b, c). These results suggested that PTEN protein is a potential inhibitor of the axon growth ability of $\mathrm{IB}^{+}{ }^{+}$neurons.

\section{Inhibition of PTEN promotes axon regeneration in $\mathrm{IB4}^{+}$sensory neurons.}

Next, to investigate the effect of PTEN on $\mathrm{IB}^{+}{ }^{+}$neuronal axon regeneration, we administrated a specific PTEN inhibitor, either $10 \mathrm{nM}$ SF1670 or $200 \mathrm{nM}$ BPV in cultured DRG neurons (Christie et al., 2010). The pS6 is a well-known downstream substrate of PTEN, and previous findings have shown that inhibition of PTEN can promote phosphorylation of pS6. In accordance with these previous findings, we found that the level of phospho-pS6 protein significantly increased in the drug treatment group (Fig. 5a). Additionally, we also found that the axon length of IB4 ${ }^{+}$neurons was much longer in the SF1670 and BPV treated groups than in the control group (Fig. 5b, c). In accordance with the pharmacological results, the axon length of the IB4 ${ }^{+}$neurons from the PTEN knockout mice was also significant increased compared to the control mice (Fig 6a, b).

\section{PTEN inhibition of peripheral axon growth is independent of the mTOR pathway}

It has been reported that the activation of the mTOR pathway can enhance axon outgrowth of adult CNS axon regeneration (Lim et al., 2016; Park et al., 2008), and that 
mTOR activity is regulated by PTEN. Therefore, to determine whether PTEN regulates sensory axon growth via mTOR activity, we simultaneously blocked both PTEN and mTOR activity using a specific pharmacological inhibitor. The adult DRG neurons were cultured for 3 days with both PTEN and mTOR inhibitors, and axon length was measured. Our data indicated that mTOR inhibition did not affect the axon growth promoting effect of the PTEN inhibitor (Fig 7a, b). There was no obvious difference in axon length between the group treated with PTEN inhibitor only and the group treated with PTEN inhibitor combined with rapamycin. This means that the regulatory effect of PTEN on adult peripheral sensory neurons is not dependent on the mTOR pathway.

\section{Discussion}

and it has been hypothesized that damaged peripheral sensory neurons can spontaneously regenerate axons. However, recent accumulating evidence has shown that only some parts of adult sensory neurons can regenerate axons after injury. Researchers have characterized multiple subgroups of adult DRG neurons according to their morphological and phenotypic differences. For example, based on their lectin Griffonia simplicifolia IB4 binding ability, adult DRG neurons can be classified as either $\mathrm{IB}^{+}{ }^{+}$or $\mathrm{IB}^{-}$. Out of these two types, $\mathrm{IB} 4^{+}$ neurons have neither p75 neurotrophin receptors, nor Tyrosine kinases (Trk) (Christie et al., 2010). Therefore, IB $4^{+}$neurons possess very limited axon regeneration abilities. It has been well established that a primary injury of adult sensory neurons, known as a "conditioning 
lesion', can prime sensory neurons into an active growth state, and dramatically increase their intrinsic axon regenerative ability. However, even a conditioning lesion cannot promote axon regeneration of $\mathrm{IB}^{+}{ }^{+}$neurons.

The specific phosphatase activity of tumor suppressor gene PTEN (Guo et al., 2002; Li et al., 1997) was first discovered in 1997. PTEN can dephosphorylate phosphatidylinositol-3,4,5-diphosphate (PIP3) to generate phosphatidylinostitol-4,5-diphosphate (PIP2). By doing so, PTEN regulates the intrinsic axon growth ability of adult retina ganglion cells (RGCs) and cortical neurons, and therefore plays a predominant role in mammalian nerve regeneration (Liu et al., 2010; Park et al., 2008). Previous findings have shown that knocking out the PTEN gene significantly promotes CNS axon regeneration in the optic nerve and in the cortical spinal tract. Additionally, PTEN gene expression increases during embryonic development, and, at the same time, the axon regeneration ability of mammalian neurons decreases with embryonic maturation. These studies clearly indicate that PTEN is a critical inhibitor of axon regeneration. Interestingly, it has been reported that IB4 sensory neurons possess high levels of PTEN proteins (Christie et al., 2010). In agreement with these previous findings, our immunohistological staining also revealed that the expression of PTEN in $\mathrm{IB}^{+}{ }^{+}$neurons was significantly higher than in IB4 ${ }^{-}$ neurons. Most adult animal DRG neurons express laminin receptor alpha7beta1 (Ekstrom et al., 2003), and its expression increases after peripheral nerve injury (Wallquist et al., 2004; Werner et al., 2000), thereby promoting axon growth on laminin (Lankford et al., 1998; Smith and Skene, 1997). However, Gardiner, et al (Gardiner et al., 2005) found that IB4 ${ }^{+}$neurons lack integrin alpha 7 and GAP43, which is a marker of axon regeneration (Benowitz and 

lesion does not alter PTEN expression. Our data also showed that the inhibition of PTEN activity with its specific pharmacological inhibitor markedly promoted IB4 neuronal axon regeneration. Furthermore, knocking out PTEN in adult sensory neurons also significantly enhances axon regeneration in vitro and in vivo, and these findings are in accordance with a previous study on CNS axon regeneration. Thus, our data clearly indicate that PTEN is a critical repressor of $\mathrm{IB}^{+}{ }^{+}$neuronal axon regeneration.

It has been reported that PTEN regulates mature CNS axon regeneration through the activation of the mammalian target of rapamycin (mTOR) pathway (Liu et al., 2010; Park et al., 2008). Our data confirmed that the deletion of PTEN can also elevate the axon regenerative ability of adult peripheral sensory neurons. Additionally, the specific pharmacological inhibition of PTEN significantly promoted axon growth in the cultured DRG neurons. However, the mTOR inhibitor did not affect the axon growth promoting effect of PTEN deletion. Additionally, the mTOR inhibitor alone did not influence axon growth of the adult sensory neurons. Therefore, the results suggest that PTEN regulates sensory neuronal axon growth independently of the mTOR pathway. PTEN also regulates Glycogen synthase kinase-3 beta (GSK3 $\beta$ ) activity independently of the mTOR pathway. Phosphorylation of GSK3 $\beta$ by PTEN-Akt is known to be the major mechanism by which GSK3 $\beta$ is inactivated. 
In conclusion, our results demonstrated that $\mathrm{IB}^{+}{ }^{+}$sensory neurons express a high level of

PTEN, and that even peripheral axotomy does not modify PTEN expression in IB4 ${ }^{+}$sensory neurons. Furthermore, we found that PTEN inhibition dramatically promotes axon growth of both $\mathrm{IB}_{4}^{+}$sensory neurons and IB4 ${ }^{-}$neurons. Taken together, these data indicate that PTEN is a key intrinsic regulator of axon growth ability of adult sensory neurons.

\section{References}

Bennett DL, Michael GJ, Ramachandran N, Munson JB, Averill S, Yan Q, McMahon SB, Priestley JV. 1998. A distinct subgroup of small DRG cells express GDNF receptor components and GDNF is protective for these neurons after nerve injury. The Journal of neuroscience : the official journal of the Society for Neuroscience 18(8):3059-3072.

Benowitz LI, Routtenberg A. 1997. GAP-43: an intrinsic determinant of neuronal development and plasticity. Trends in neurosciences 20(2):84-91.

Bhagat S, Vandana S, Anand K, Kurien K, Martinez JA, Chu C, Chris A, Zochodne DW. 2014. Regeneration of diabetic axons is enhanced by selective knockdown of the PTEN gene. Brain A Journal of Neurology 137(4):1051-1067.

Christie KJ, Webber CA, Martinez JA, Singh B, Zochodne DW. 2010. PTEN inhibition to facilitate intrinsic regenerative outgrowth of adult peripheral axons. The Journal of neuroscience : the official journal of the Society for Neuroscience 30(27):9306-9315.

Danilov CA, Steward O. 2015. Conditional genetic deletion of PTEN after a spinal cord injury enhances regenerative growth of CST axons and motor function recovery in 
mice. Experimental neurology 266:147-160.

Du K, Zheng S, Zhang Q, Li S, Gao X, Wang J, Jiang L, Liu K. 2015. Pten Deletion Promotes Regrowth of Corticospinal Tract Axons 1 Year after Spinal Cord Injury. The Journal of neuroscience : the official journal of the Society for Neuroscience 35(26):9754-9763.

Ekstrom PA, Mayer U, Panjwani A, Pountney D, Pizzey J, Tonge DA. 2003. Involvement of alpha7beta1 integrin in the conditioning-lesion effect on sensory axon regeneration. Molecular and cellular neurosciences 22(3):383-395.

Gardiner NJ, Fernyhough P, Tomlinson DR, Mayer U, von der Mark H, Streuli CH. 2005. Alpha7 integrin mediates neurite outgrowth of distinct populations of adult sensory neurons. Molecular and cellular neurosciences 28(2):229-240.

Guo F, A EMP, Christian M, Andreas L, Norbert S, Knut D, Peter M. 2002. Reduced PTEN expression in gastric cancer and in the gastric mucosa of gastric cancer relatives. European Journal of Gastroenterology and Hepatology 14(3).

Kurimoto T, Yin Y, Omura K, Gilbert HY, Kim D, Cen LP, Moko L, Kugler S, Benowitz LI. 2010. Long-distance axon regeneration in the mature optic nerve: contributions of oncomodulin, cAMP, and pten gene deletion. The Journal of neuroscience : the official journal of the Society for Neuroscience 30(46):15654-15663.

Lankford KL, Waxman SG, Kocsis JD. 1998. Mechanisms of enhancement of neurite regeneration in vitro following a conditioning sciatic nerve lesion. The Journal of comparative neurology 391(1):11-29.

Leclere PG, Norman E, Groutsi F, Coffin R, Mayer U, Pizzey J, Tonge D. 2007. Impaired axonal regeneration by isolectin B4-binding dorsal root ganglion neurons in vitro. The 
Journal of neuroscience : the official journal of the Society for Neuroscience 27(5):1190-1199.

Lee JK, Geoffroy CG, Chan AF, Tolentino KE, Crawford MJ, Leal MA, Kang B, Zheng B. 2010. Assessing spinal axon regeneration and sprouting in Nogo-, MAG-, and OMgp-deficient mice. Neuron 66(5):663-670.

Li J, Yen C, Liaw D, Podsypanina K, Bose S, Wang SI, Puc J, Miliaresis C, Rodgers L, McCombie R, Bigner SH, Giovanella BC, Ittmann M, Tycko B, Hibshoosh H, Wigler MH, Parsons R. 1997. PTEN, a putative protein tyrosine phosphatase gene mutated in human brain, breast, and prostate cancer. Science 275(5308):1943-1947.

Lim JH, Stafford BK, Nguyen PL, Lien BV, Wang C, Zukor K, He Z, Huberman AD. 2016. Neural activity promotes long-distance, target-specific regeneration of adult retinal axons. Nature neuroscience 19(8):1073-1084.

Liu K, Lu Y, Lee JK, Samara R, Willenberg R, Sears-Kraxberger I, Tedeschi A, Park KK, Jin D, Cai B, Xu B, Connolly L, Steward O, Zheng B, He Z. 2010. PTEN deletion enhances the regenerative ability of adult corticospinal neurons. Nature neuroscience 13(9):1075-1081.

Ning K, Drepper C, Valori CF, Ahsan M, Wyles M, Higginbottom A, Herrmann T, Shaw P, Azzouz M, Sendtner M. 2010. PTEN depletion rescues axonal growth defect and improves survival in SMN-deficient motor neurons. Human molecular genetics 19(16):3159-3168.

Ohtake Y, Hayat U, Li S. 2015. PTEN inhibition and axon regeneration and neural repair. Neural regeneration research 10(9):1363-1368. 
Park KK, Liu K, Hu Y, Kanter JL, He Z. 2010. PTEN/mTOR and axon regeneration. Experimental neurology 223(1):45-50.

Park KK, Liu K, Hu Y, Smith PD, Wang C, Cai B, Xu B, Connolly L, Kramvis I, Sahin M, He Z. 2008. Promoting axon regeneration in the adult CNS by modulation of the PTEN/mTOR pathway. Science 322(5903):963-966.

Saijilafu, Hur EM, Liu CM, Jiao Z, Xu WL, Zhou FQ. 2013. PI3K-GSK3 signalling regulates mammalian axon regeneration by inducing the expression of Smad1. Nature communications 4:2690.

Smith DS, Skene JH. 1997. A transcription-dependent switch controls competence of adult neurons for distinct modes of axon growth. The Journal of neuroscience : the official journal of the Society for Neuroscience 17(2):646-658.

Tucker BA, Rahimtula M, Mearow KM. 2006. Laminin and growth factor receptor activation stimulates differential growth responses in subpopulations of adult DRG neurons. The European journal of neuroscience 24(3):676-690.

Wallquist W, Zelano J, Plantman S, Kaufman SJ, Cullheim S, Hammarberg H. 2004. Dorsal root ganglion neurons up-regulate the expression of laminin-associated integrins after peripheral but not central axotomy. The Journal of comparative neurology 480(2):162-169.

Werner A, Willem M, Jones LL, Kreutzberg GW, Mayer U, Raivich G. 2000. Impaired axonal regeneration in alpha7 integrin-deficient mice. The Journal of neuroscience : the official journal of the Society for Neuroscience 20(5):1822-1830. 
Figure legends

Figure 1. Injury induces down regulation of PTEN expression in adult sensory neurons.

(a) Representative Western Blot images of PTEN expression in L4-L5 DRGs of adult mice 3 days after sciatic nerve axotomy. Compared to uninjured control, PTEN protein level is markedly down-regulated by peripheral axotomy $(n=3)$.

(b) Immunohistological staining showed PTEN expression was decreased in the L4 DRG tissues after sciatic nerve axotomy. Green: PTEN, Scale bar: $100 \mu \mathrm{m}$.

(c) Quantification of PTEN mRNA levels by qPCR. The relative level of PTEN mRNA expression in the L4 DRGs was reduced 3 days after sciatic nerve axotomy $(\mathrm{n}=3)$. $* P<0.05$.

\section{Figure 2. PTEN inhibition promotes sensory axon regeneration in vitro and in vivo.}

(a) Adult DRG neurons were cultured in vitro for 3 days, and treated with PTEN inhibitor SF1670 (1nM or $10 \mathrm{nM})$ or BPV (200 nM). The vehicle DMSO was as control. All neurons were stained with anti- $\beta$ III tubulin (green). Scale bar: $100 \mu \mathrm{m}$. Quantification of the average length of the longest axons $(n=3)$.

(b) The pharmacological inhibition of PTEN activity promoted the axon growth of peripheral sensory neurons $(\mathrm{n}=3) . * P<0.05, \quad * * * P<0.001$.

(c) The pharmacological inhibition of PTEN activity markedly increases phospho-S6 expression, which is a well-known downstream target of the PTEN, in the cultured DRG neurons.

(d) The adult DRG neurons were cultured from the Advilin-Cre induced PTEN knockout mice. Scale bar: $100 \mu \mathrm{m}$.

(e) Axon length of adult DRG neuron in the PTEN knockout mice was significantly longer 
than the PTEN ${ }^{\text {flox/flox }}$ mice $(\mathrm{n}=3)$. *** $P<0.001$.

(f) The left L3-L4 DRGs were electroporated with EGFP plasmid and sciatic nerve was crushed two days after electroporation. Another three days later, whole sciatic nerve segment was harvested. Red dot line is crush site. Arrow head is regenerating axons. Scale bar: $500 \mu \mathrm{m}$.

(g) PTEN knockout significantly promotes sciatic nerve axon regeneration in vivo $(\mathrm{n}=5)$. *** $P<0.001$.

Figure 3. $\mathrm{IB4}^{+}$neurons possess higher PTEN expression, and lower intrinsic axon growth ability.

(a) Immunohistological staining of L4 DRG section showed that IB4+ neurons possess higher PTEN expression. Scale bar, $100 \mu \mathrm{m}$.

(b) Adult DRG neurons were cultured for three days, and stained with anti- $\beta$ III tubulin (red) and Alexa Fluor 488-conjugated Isolectin IB4 (green). Scale bar, 100 $\mu \mathrm{m}$.

(c) Axon length from IB4 ${ }^{+}$neurons was significantly shorter than IB4- neuron $(\mathrm{n}=3){ }^{* * *} P$ $<0.001$.

\section{Figure 4. PTEN expression in IB4+ neurons was not affected by sciatic nerve axotomy.}

(a) Immunohistostaining of L4 DRG section showed that PTEN expression in IB4 ${ }^{+}$neurons was not affected by sciatic nerve axotomy. Arrowhead : IB4 ${ }^{+}$neurons. Scale bar, $100 \mu \mathrm{m}$.

(b) Adult DRG neurons were culture one day after peripheral nerve axotomy, and stained for anti- $\beta$ III tubulin (red) and Alexa Fluor 488-conjugated Isolectin IB4 (green). Scale bar, $100 \mu \mathrm{m}$.

(c) IB4- neuronal axon growth is significantly enhanced by peripheral axotomy, however, intrinsic axon growth ability of IB $4^{+}$neuron is not affected $(\mathrm{n}=3)$. *** $P<0.001$. 
437

438

439

440

441

442

443

444

445

446

447

448

449

450

451

452

453

454

455

456

457

458

Figure 5. PTEN inhibition dramatically promotes $\mathrm{IB4}^{+}$neuronal axon growth.

(a) Representative western blot images of phosphor-S6 in adult DRGs neuronal culture with PTEN inhibitor SF1670 or BPV, and vehicle DMSO were as control group.

(b) Adult DRG neurons were cultured for three days with PTEN inhibitor SF1670 (10 nM ) or BPV (200 nM ) treatment. Vehicle (DMSO) was as control. All neurons were stained with anti-ßIII tubulin (red) and Alexa Fluor 488-conjugated Isolectin IB4 (green). Scale bar, $100 \mu \mathrm{m}$.

(c) Administration of PTEN inhibitor significantly increases axon length of IB4+ neuron $(\mathrm{n}=3) . * P<0.05, \quad * * P<0.01$.

Figure 6. Knockout of PTEN gene promotes $\mathrm{IB4}^{+}$neuronal axon growth.

(a) Adult DRG sensory neurons were culture for three days from advilin-cre induced PTEN knockout mice. All neurons were stained with anti- $\beta$ III tubulin (red) and Alexa Fluor 488-conjugated Isolectin IB4 (green). Scale bar, $100 \mu \mathrm{m}$.

(b) Quantification of the average axon length showed that PTEN knockout markedly promotes

IB4 ${ }^{+}$neuronal axon growth $(\mathrm{n}=3) . * * P<0.01$.

Figure 7. PTEN inhibition induced peripheral axon growth is independent of the mTOR pathway.

(a) Adult DRG neurons were cultured with rapamycin (20nM) only, or cultured with both rapamycin 20nM and PTEN inhibitor (10nM SF1670 or 200nM BPV). All neurons were stained with anti- $\beta$ III tubulin (green). Scale bar, $100 \mu \mathrm{m}$.

(b) Quantification of the average axon length showed that mTOR inhibition did not affect the 
bioRxiv preprint doi: https://doi.org/10.1101/2020.04.02.019042; this version posted April 3, 2020. The copyright holder for this preprint (which was not certified by peer review) is the author/funder. All rights reserved. No reuse allowed without permission.

459

axon growth promoting effect of the PTEN inhibitor (n=3). ** $P<0.01$.

460 


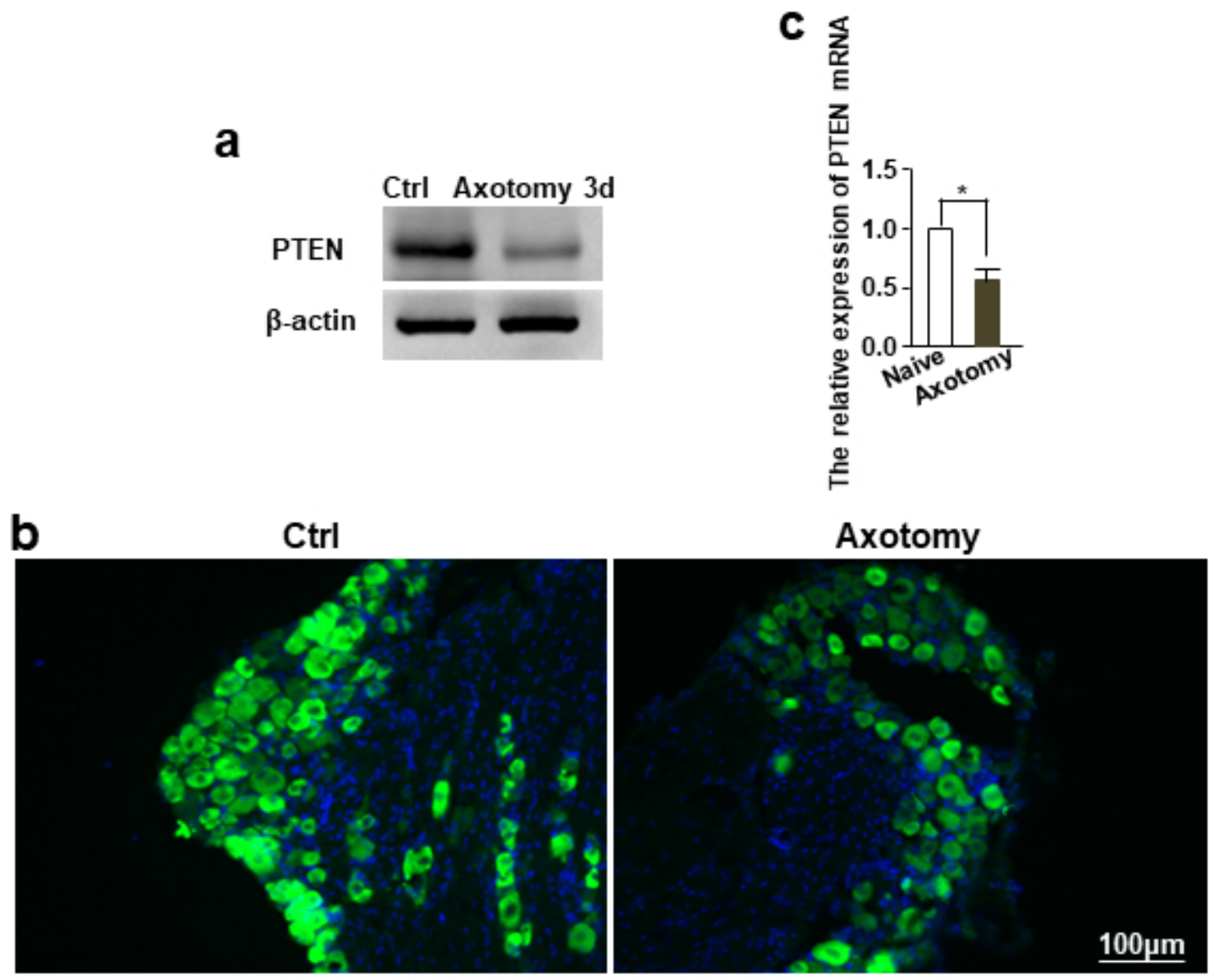

Figure 1 

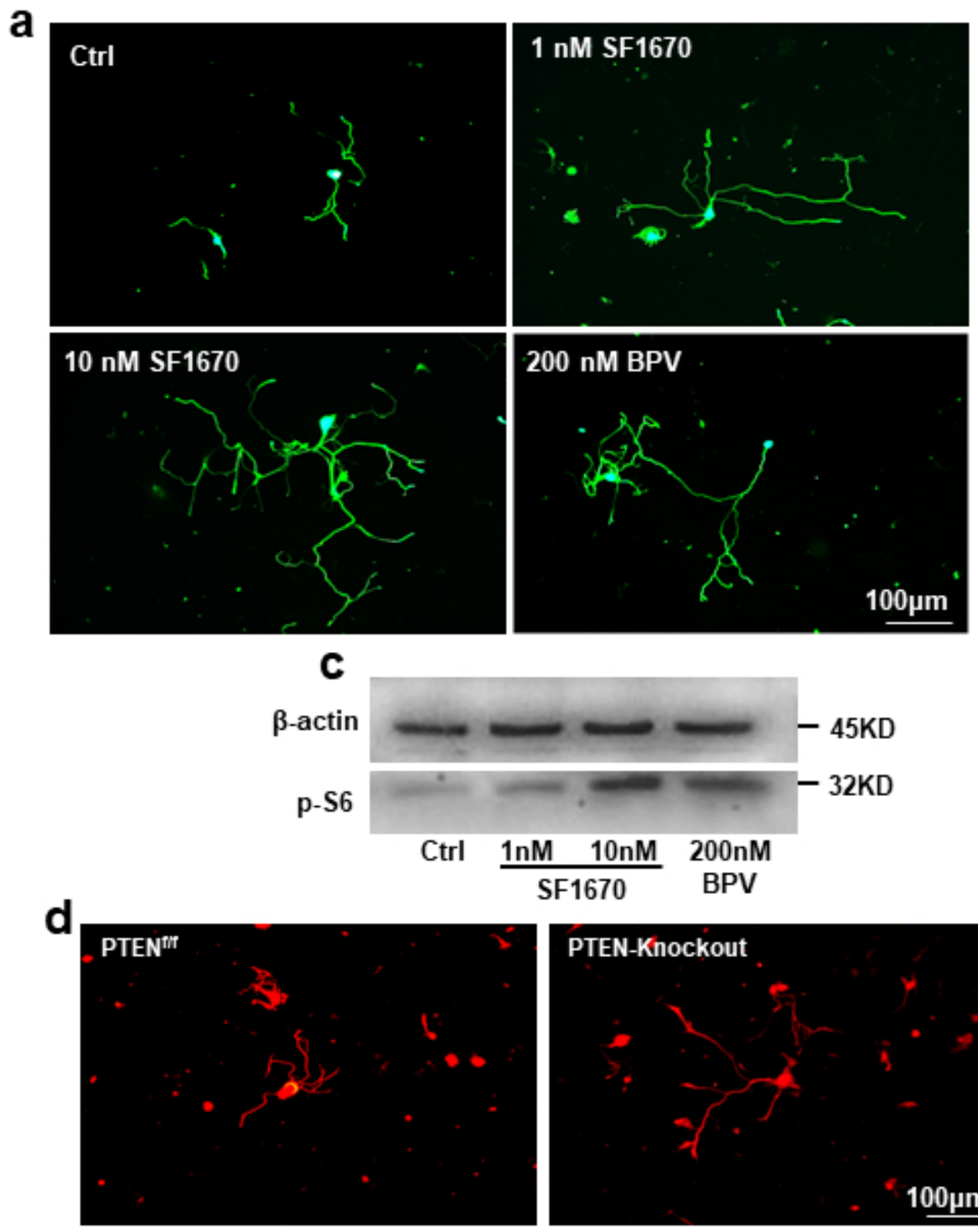

PTEN-knockout

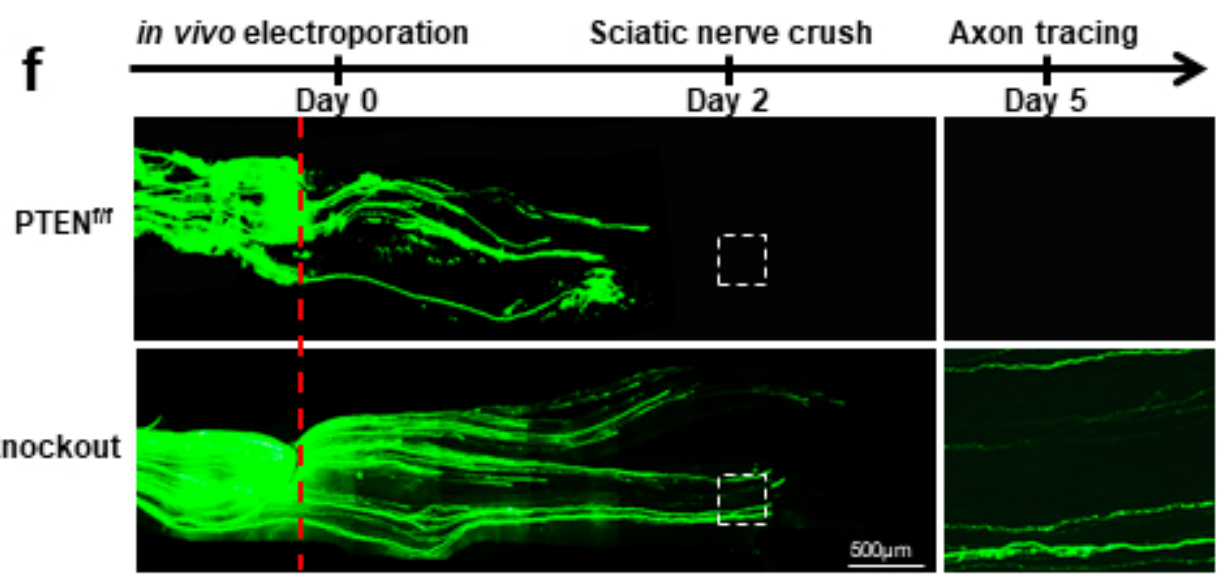

b

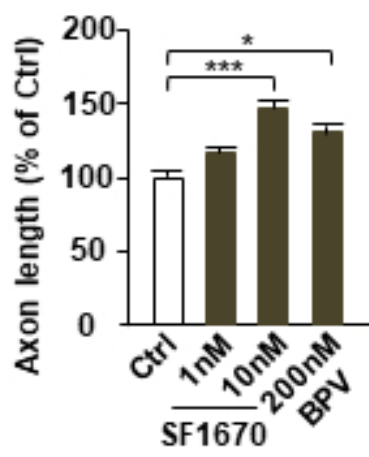

e
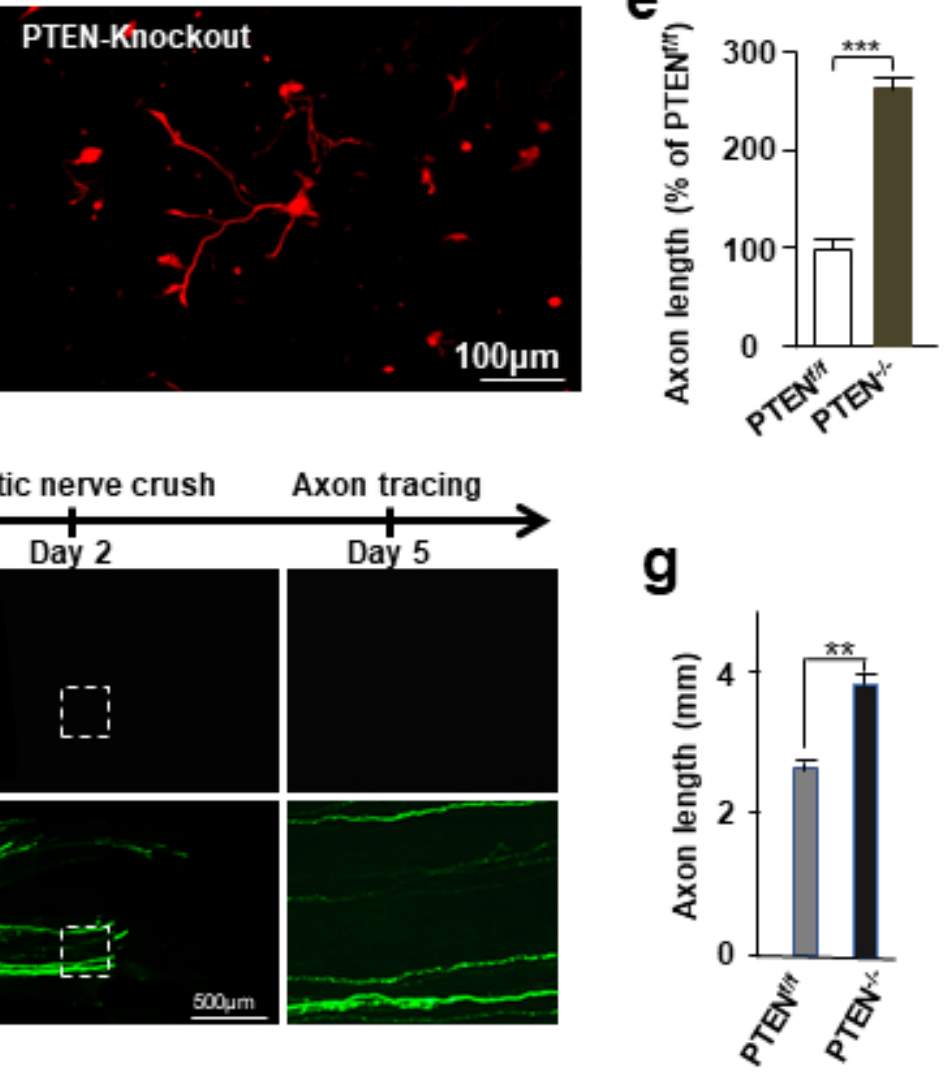

\section{Figure 2}



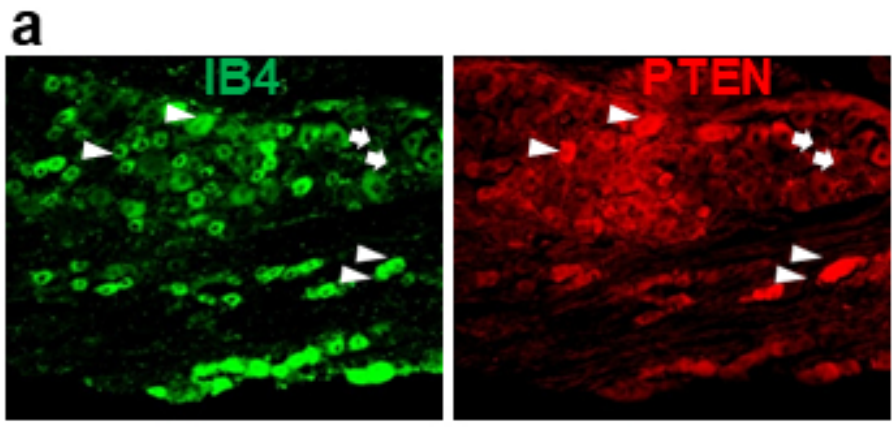

IB4+神经元

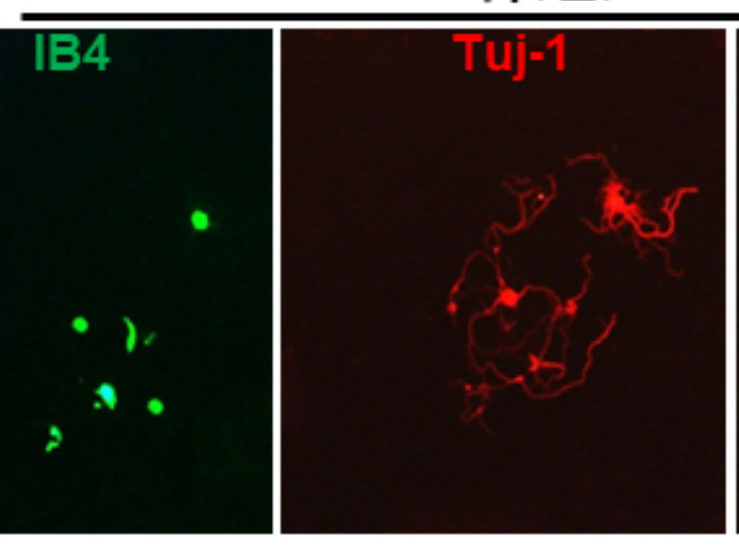

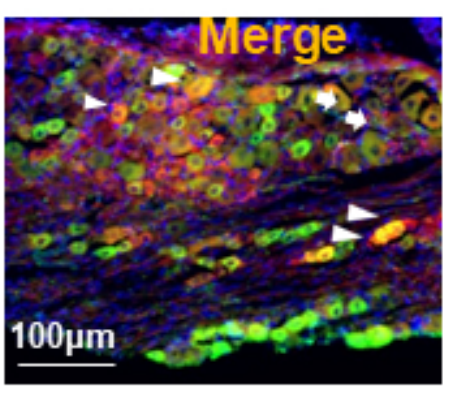

C

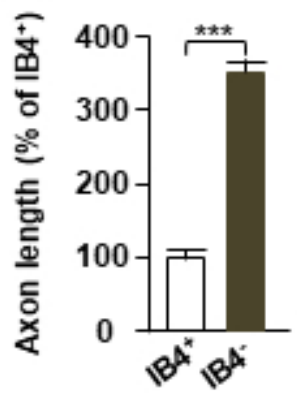

IB4 - 神经元
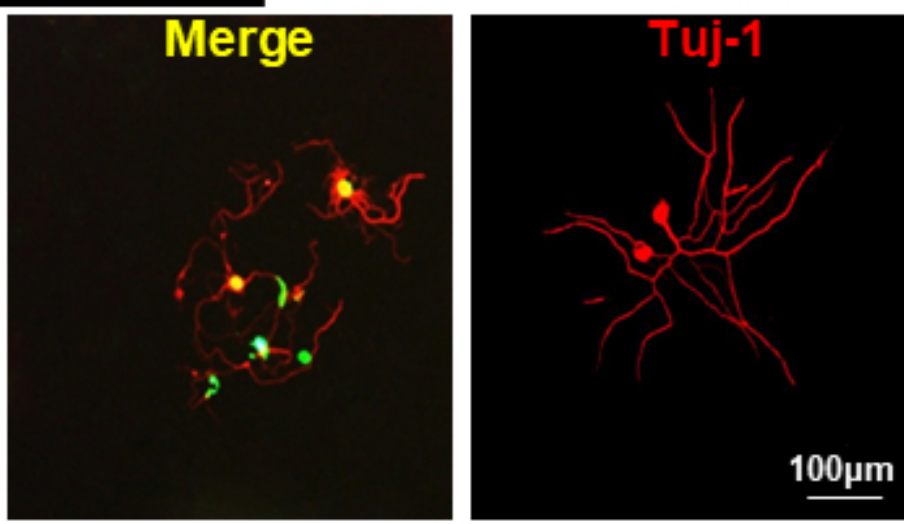

Figure 3 

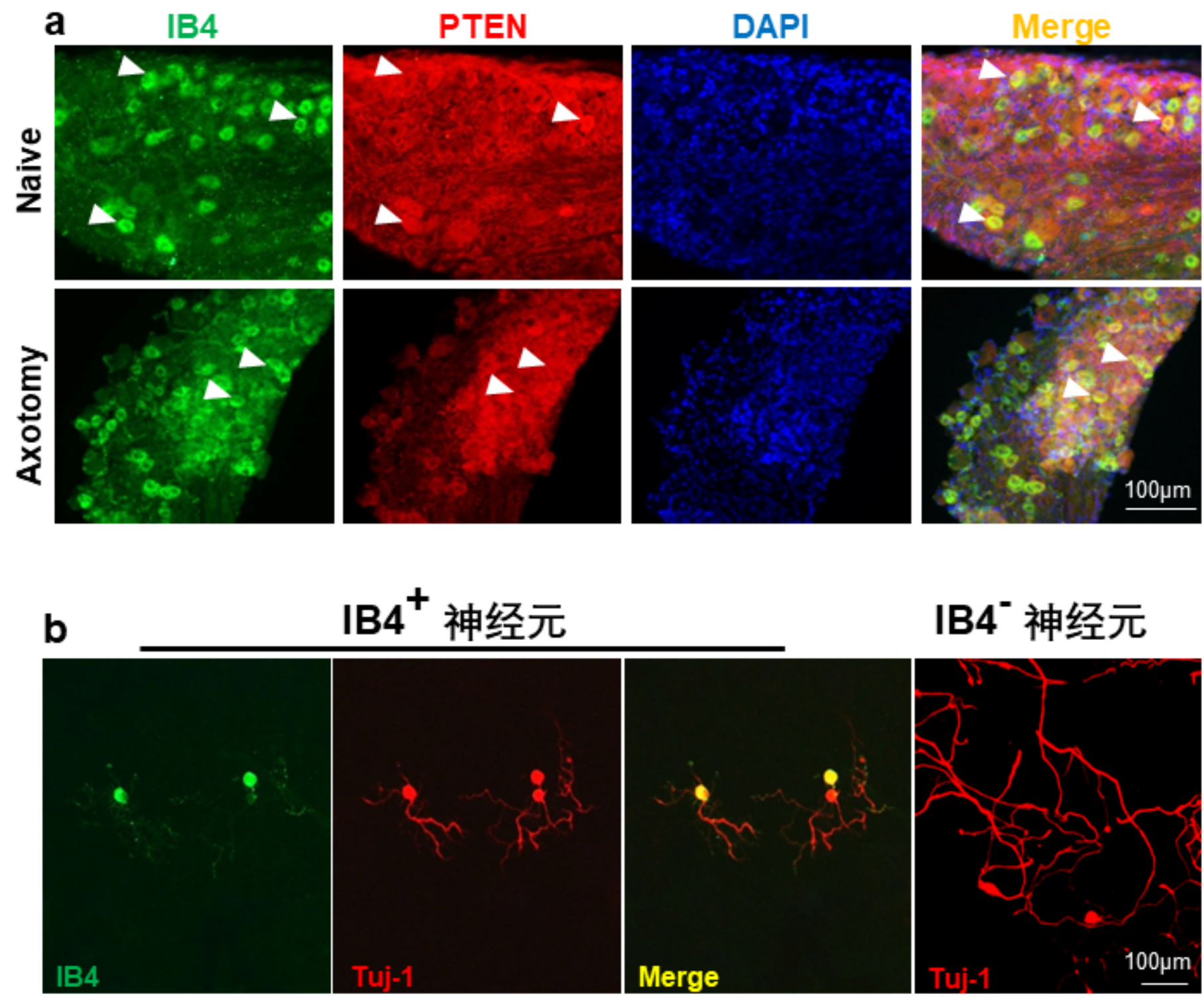

IB4 ${ }^{-}$神经元

C

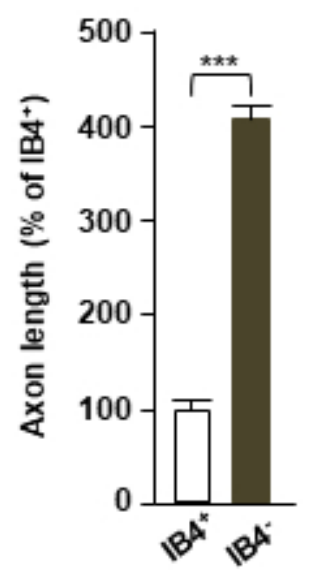

Figure 4

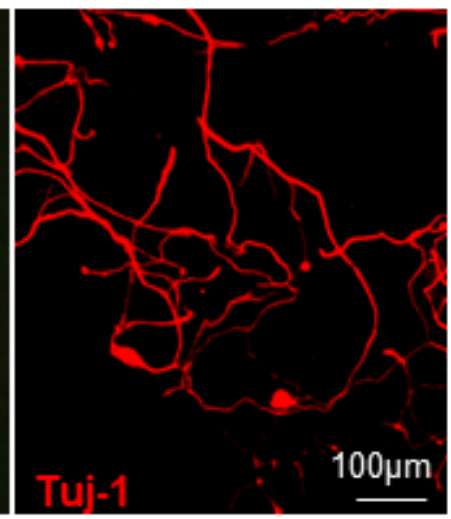

$100 \mu \mathrm{m}$

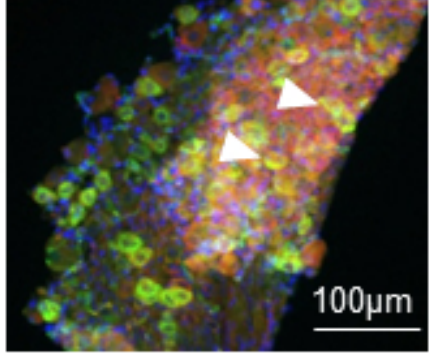




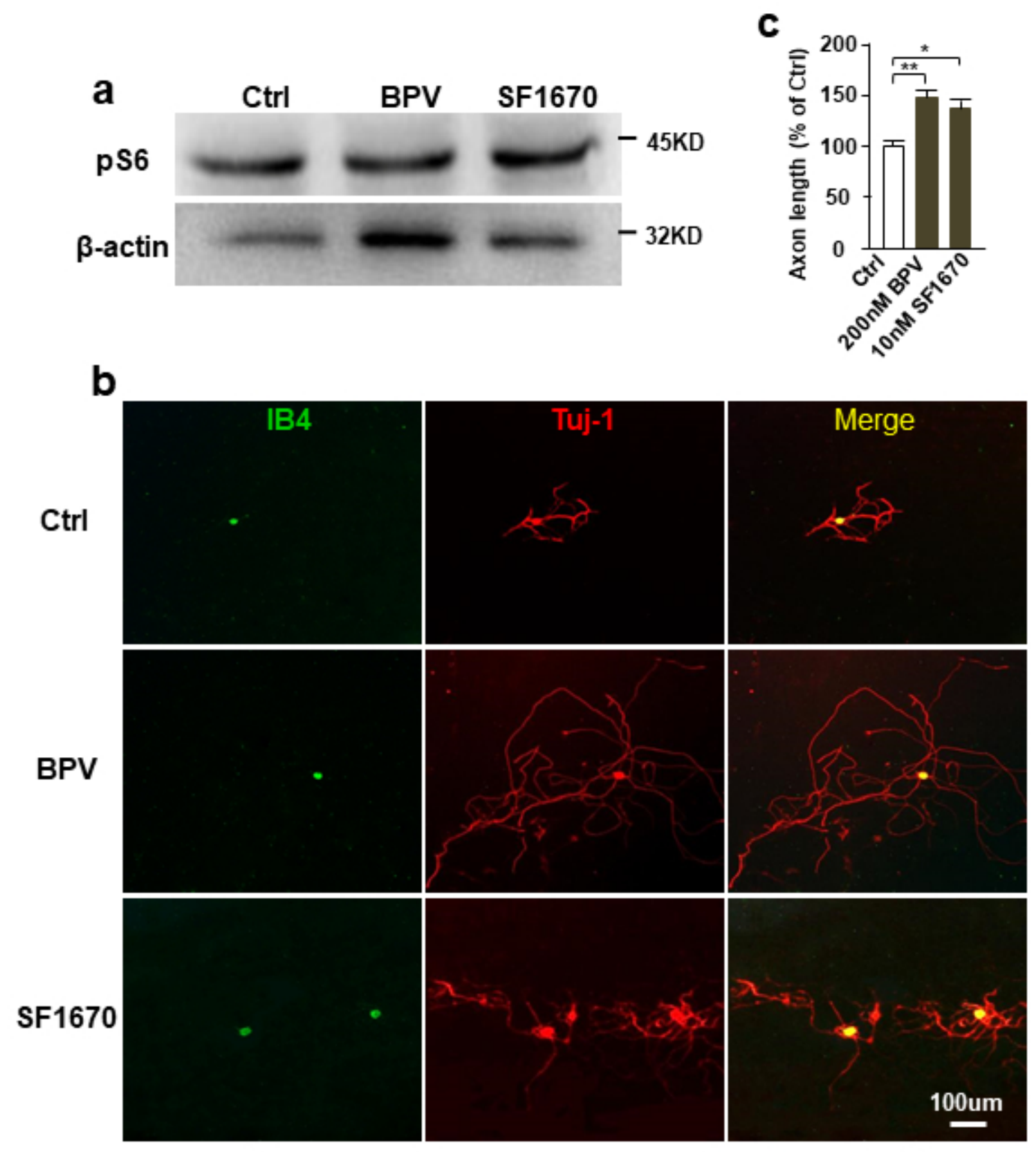

Figure 5 


\section{a}

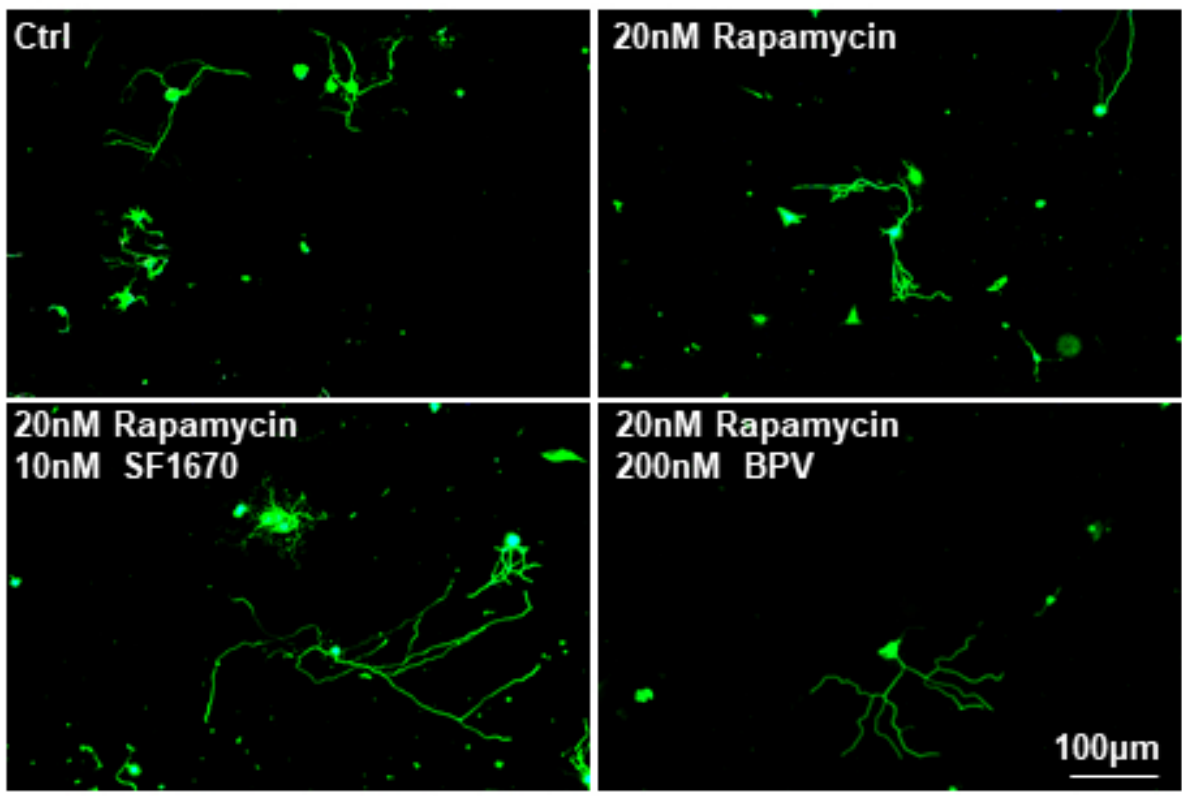

b

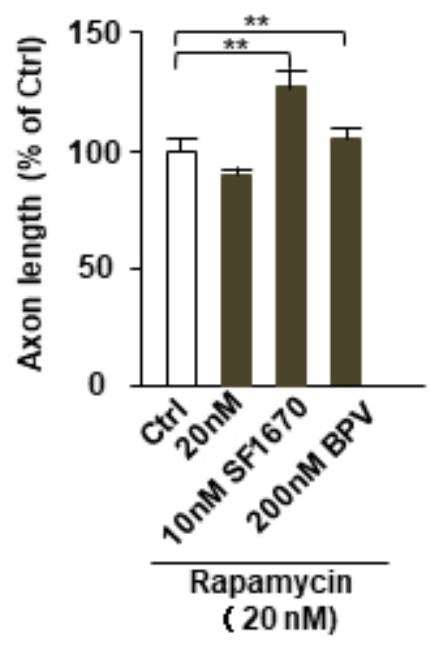

Figure 7 